\title{
Time-Fractional Heat Conduction in a Half-Line Domain due to Boundary Value of Temperature Varying Harmonically in Time
}

\begin{abstract}
Yuriy Povstenko
Institute of Mathematics and Computer Science, Faculty of Mathematics and Natural Sciences, Jan Dlugosz University in Czestochowa, Armii Krajowej 13/15, 42-200 Czestochowa, Poland

Correspondence should be addressed to Yuriy Povstenko; j.povstenko@ajd.czest.pl

Received 23 August 2016; Accepted 23 October 2016

Academic Editor: Filippo de Monte

Copyright (C) 2016 Yuriy Povstenko. This is an open access article distributed under the Creative Commons Attribution License, which permits unrestricted use, distribution, and reproduction in any medium, provided the original work is properly cited.

The Dirichlet problem for the time-fractional heat conduction equation in a half-line domain is studied with the boundary value of temperature varying harmonically in time. The Caputo fractional derivative is employed. The Laplace transform with respect to time and the sin-Fourier transform with respect to the spatial coordinate are used. Different formulations of the considered problem for the classical heat conduction equation and for the wave equation describing ballistic heat conduction are discussed.
\end{abstract}

\section{Introduction}

In the paper [1] and later on in the book [2] Nowacki studied the classical parabolic heat conduction equation with a heat source term varying harmonically as a function of time

$$
\frac{\partial T(x, t)}{\partial t}=a \frac{\partial^{2} T(x, t)}{\partial x^{2}}+q_{0} \delta(x) e^{i \omega t}
$$

in the domain $-\infty<x<\infty$. Here $a>0$ is the thermal diffusivity coefficient, $\delta(x)$ is the Dirac delta function, and $\omega>0$ denotes the frequency.

Nowacki's solution of (1) is based on the assumption that temperature can be expressed as a product of the auxiliary function $U(x)$ and the time harmonic term

$$
T(x, t)=U(x) e^{i \omega t} .
$$

In this case, there are no initial and boundary conditions (excepting the zero condition at $x \rightarrow \pm \infty$ ), and the problem is reduced to solving the corresponding equation for the auxiliary function $U(x)$. The final result reads

$$
T(x, t)=\frac{q_{0}}{2 a \sqrt{i \omega / a}} e^{-|x| \sqrt{i \omega / a}+i \omega t} .
$$

The square root of the imaginary unit is defined as $\sqrt{i}=e^{i \pi / 4}$.
If the heat conduction equation

$$
\frac{\partial T(x, t)}{\partial t}=a \frac{\partial^{2} T(x, t)}{\partial x^{2}}
$$

is considered in a half-line domain $0<x<\infty$, then the boundary condition at $x=0$ should be imposed. For example, we can assume the Dirichlet boundary condition varying harmonically in time:

$$
T(x, t)=T_{0} e^{i \omega t} \quad x=0 .
$$

Similar analysis can be also carried out in the case of the boundary value of heat flux varying harmonically in time (the physical Neumann boundary condition). Boundary conditions varying harmonically in time describe various situations, in particular, thermal processing of materials using pulsed lasers or collection of solar energy [3].

Under Nowacki's assumption (2), there is no initial condition, and for the auxiliary function $U(x)$ we obtain

$$
\begin{aligned}
i \omega U(x) & =a \frac{d^{2} U(x)}{d x^{2}}, \quad 0<x<\infty, \\
U(x) & =T_{0} \quad x=0, \\
\lim _{x \rightarrow \infty} U(x) & =0 .
\end{aligned}
$$


To compare with the subsequent results it is worthwhile to solve (6) under boundary conditions (7) and (8) using the sin-Fourier transform with respect to the spatial coordinate $x$. The solution has a form

$$
U(x)=T_{0} e^{-x \sqrt{i \omega / a}}
$$

Hence,

$$
T(x, t)=T_{0} e^{-x \sqrt{i \omega / a}+i \omega t}
$$

If the surface temperature is described by the dependence

$$
T(x, t)=T_{0} \sin (\omega t) \quad x=0,
$$

then the solution becomes [3]

$$
T(x, t)=T_{0} \exp \left(-x \sqrt{\frac{\omega}{2 a}}\right) \sin \left(\omega t-x \sqrt{\frac{\omega}{2 a}}\right) .
$$

Many experimental and theoretical investigations testify that in media with complex internal structure the standard heat conduction equation is no longer sufficiently accurate. This results in formulation of nonclassical theories, in which the parabolic heat conduction equation is replaced by more general one (see [4-12] and the references therein).

For example, Green and Naghdi [7] proposed the theory of thermoelasticity without energy dissipation based on the wave equation for temperature. In the framework of this theory, the following boundary value problem can be studied:

$$
\begin{aligned}
\frac{\partial^{2} T(x, t)}{\partial t^{2}} & =a \frac{\partial^{2} T(x, t)}{\partial x^{2}}, \\
T(x, t) & =T_{0} e^{i \omega t} \quad x=0 .
\end{aligned}
$$

Under the assumption (2), using the sin-Fourier transform, we get the solution (see (A.3) from appendix)

$$
T(x, t)=T_{0} \cos \left(\frac{\omega x}{\sqrt{a}}\right) e^{i \omega t} .
$$

\section{Time-Fractional Heat Conduction}

The time-nonlocal generalization of the Fourier law with the "long-tail" power kernel [11, 13-15] can be interpreted in terms of fractional calculus (theory of integrals and derivatives of noninteger order) and results in the timefractional heat conduction equation

$$
{ }^{C} D_{0+}^{\alpha} T=a \Delta T, \quad 0<\alpha \leq 2,
$$

with the Caputo fractional derivative of order $\alpha$ defined as [16-18]

$$
\begin{array}{r}
{ }^{C} D_{0+}^{\alpha} f(t)=\frac{1}{\Gamma(n-\alpha)} \int_{0}^{t}(t-\tau)^{n-\alpha-1} \frac{d^{n} f(\tau)}{d \tau^{n}} d \tau, \\
n-1<\alpha<n,
\end{array}
$$

and having the following Laplace transform rule:

$$
\begin{array}{r}
L\left\{{ }^{C} D_{0+}^{\alpha} f(t)\right\}=s^{\alpha} f^{*}(s)-\sum_{k=0}^{n-1} f^{(k)}\left(0^{+}\right) s^{\alpha-1-k}, \\
n-1<\alpha<n,
\end{array}
$$

where the asterisk denotes the transform, $s$ is the transform variable, and $\Gamma(x)$ is the gamma function. The Caputo fractional derivative is a regularization in the time origin for the Riemann-Liouville fractional derivative by incorporating the relevant initial conditions [19]. The major utility of the Caputo fractional derivative is caused by the treatment of differential equations of fractional order for physical applications, where the initial conditions are usually expressed in terms of a given function and its derivatives of integer (not fractional) order, even if the governing equation is of fractional order $[17,20]$. Additional discussion on the use of the Caputo and Riemann-Liouville fractional derivatives can be found in [21] (see Section 3.4 "Which type of fractional derivative? Caputo or Riemann-Liouville?" in this book).

Equations with fractional derivatives describe many important physical phenomena in amorphous, colloid, glassy, and porous materials, in fractals, comb structures, polymers, and random and disordered materials, in viscoelasticity and hereditary mechanics of solids, in biological systems, and in geophysical and geological processes (see, e.g., [2230] and the references therein). Important applications of fractional calculus can be found in such fields as fractional dynamics [31-35], fractional kinetics [36-38], and fractional thermoelasticity $[11,12,39-41]$.

Equation (15) describes the whole spectrum from localized heat conduction (the Helmholtz equation for $\alpha \rightarrow 0$ ) through the standard heat conduction $(\alpha=1)$ to the ballistic heat conduction (the wave equation when $\alpha=2$ ).

The interested reader is referred to the book [15], which systematically presents solutions to different initial and boundary value problems for the time-fractional diffusionwave equation (15) in Cartesian, cylindrical, and spherical coordinates. In $[42,43]$, this equation was considered in unbounded domains with the source term varying harmonically in time.

In the present paper, we study the Dirichlet problem for the time-fractional heat conduction equation in a halfline domain with the surface value of temperature varying harmonically in time. The integral transform technique is used. Different formulations of the considered problem for the classical heat conduction equation $(\alpha=1)$ and for the wave equation describing ballistic heat conduction $(\alpha=2)$ are discussed.

\section{Formulation of the Problem}

We consider the time-fractional heat conduction equation in a half-line:

$$
\begin{aligned}
& { }^{C} D_{0+}^{\alpha} T=a \frac{\partial^{2} T(x, t)}{\partial x^{2}} \\
& \quad 0<x<\infty, 0<t<\infty, 0<\alpha \leq 2,
\end{aligned}
$$


under the harmonic boundary condition

$$
T(x, t)=T_{0} e^{i \omega t} \quad x=0,
$$

and zero condition at infinity

$$
\lim _{x \rightarrow \infty} T(x, t)=0 \text {. }
$$

For the Caputo derivative of the exponential function we have

$$
\begin{aligned}
{ }^{C} D_{0+}^{\alpha} e^{\lambda t}= & \frac{1}{\Gamma(n-\alpha)} \int_{0}^{t}(t-\tau)^{n-\alpha-1} \frac{d^{n} e^{\lambda t}}{d \tau^{n}} d \tau \\
= & \frac{1}{\Gamma(n-\alpha)} \lambda^{n} \int_{0}^{t}(t-\tau)^{n-\alpha-1} e^{\lambda t} d \tau, \\
& n-1<\alpha<n .
\end{aligned}
$$

Substituting $t-\tau=\lambda^{-1} u$ (with $\lambda>0$ ) gives the final result

$$
{ }^{C} D_{0+}^{\alpha} e^{\lambda t}=\lambda^{\alpha} e^{\lambda t} \frac{\gamma(n-\alpha, \lambda t)}{\Gamma(n-\alpha)}, \quad n-1<\alpha<n,
$$

where $\gamma(a, x)$ is the incomplete gamma function [44]:

$$
\gamma(a, x)=\int_{0}^{x} e^{-t} t^{a-1} d t
$$

Hence, for fractional (noninteger) values of the order $\alpha$ of derivative,

$$
{ }^{C} D_{0+}^{\alpha} e^{\lambda t} \neq \lambda^{\alpha} e^{\lambda t}
$$

Therefore, the Nowacki assumption (2) cannot be used for the time-fractional heat conduction equation and the corresponding initial conditions should be imposed (see also $[42,43])$. In the present paper we assume zero initial conditions:

$$
\begin{aligned}
& 0<\alpha \leq 2 \quad t=0, T=0 \\
& 1<\alpha \leq 2 \quad t=0, \quad \frac{\partial T}{\partial t}=0
\end{aligned}
$$

\section{Solution to the Problem}

Application of the Laplace transform with respect to time $t$ and the sin-Fourier transform with respect to the spatial coordinate $x$ to (18) under the initial conditions (25) and the boundary conditions (19), (20) gives

$$
\widetilde{T}^{*}(\xi, s)=a T_{0} \frac{\xi}{s^{\alpha}+a \xi^{2}} \frac{1}{s-i \omega},
$$

where the tilde denotes the sin-Fourier transform and $\xi$ is the transform variable.

At first, we analyze the standard heat conduction equation corresponding to $\alpha=1$ :

$$
\widetilde{T}^{*}(\xi, s)=a T_{0} \frac{\xi}{s+a \xi^{2}} \frac{1}{s-i \omega} .
$$

The inverse Laplace transform gives (see (A.6) from appendix):

$$
\widetilde{T}(\xi, t)=a T_{0} \frac{\xi}{a \xi^{2}+i \omega}\left(e^{i \omega t}-e^{-a \xi^{2} t}\right) .
$$

Integrals (A.2) and (A.4) allow us to invert the sin-Fourier transform and to obtain the solution

$$
\begin{aligned}
T(x, t)= & T_{0} e^{-x \sqrt{i \omega / a}+i \omega t} \\
& -\frac{T_{0}}{2} e^{-x \sqrt{i \omega / a}+i \omega t} \operatorname{erfc}\left(\sqrt{i \omega t}-\frac{x}{2 \sqrt{a t}}\right) \\
& +\frac{T_{0}}{2} e^{x \sqrt{i \omega / a}+i \omega t} \operatorname{erfc}\left(\sqrt{i \omega t}+\frac{x}{2 \sqrt{a t}}\right) .
\end{aligned}
$$

The first term in (29) coincides with solution (10) and describes the quasi-steady-state oscillations; the second and third ones describe the transient process.

Starting in (27) from the inversion of the sin-Fourier transform, we get

$$
T^{*}(x, s)=T_{0} \frac{1}{s-i \omega} \exp \left(-\frac{x}{\sqrt{a}} \sqrt{s}\right) .
$$

Taking into account (A.10) and using the convolution theorem for the Laplace transform allow us to obtain an alternative form of the solution to the standard heat conduction equation:

$$
T(x, t)=\frac{T_{0} x}{2 \sqrt{\pi a}} \int_{0}^{t} \frac{1}{\tau^{3 / 2}} \exp \left(-\frac{x^{2}}{4 a \tau}\right) e^{i \omega(t-\tau)} .
$$

Another particular case of solution (26) in the transform domain corresponds to the ballistic heat conduction $(\alpha=2)$ :

$$
\widetilde{T}^{*}(\xi, s)=a T_{0} \frac{\xi}{s^{2}+a \xi^{2}} \frac{1}{s-i \omega} .
$$

Inversion of the sin-Fourier transform (see (A.2)) results in

$$
T^{*}(x, s)=T_{0} \frac{1}{s-i \omega} \exp \left(-\frac{x}{\sqrt{a}} s\right) .
$$

Taking into account (A.9), we obtain

$$
T(x, t)= \begin{cases}T_{0} e^{i \omega(t-x / \sqrt{a})}, & 0 \leq x<\sqrt{a} t, \\ 0, & \sqrt{a} t<x<\infty .\end{cases}
$$

It should be noted that solution (34) describes the wavefront at $x=\sqrt{a} t$.

In applications there often appears the value $\alpha=1 / 2$ (see investigations of diffusion on fractals [45] and comb structures $[46,47])$. Using (A.7), we get

$$
\begin{aligned}
& T(x, t)=\frac{2 a T_{0}}{\pi} \int_{0}^{\infty} \frac{\xi \sin (x \xi)}{a^{2} \xi^{4}-i \omega}\left[a \xi^{2} e^{i \omega t}\right. \\
& +\sqrt{-i \omega} e^{i \omega t} \operatorname{erfi}(\sqrt{-i \omega t}) \\
& \left.\quad-a \xi^{2} e^{a^{2} \xi^{4} t} \operatorname{erfc}\left(a \xi^{2} \sqrt{t}\right)\right] d \xi
\end{aligned}
$$


Now we return to the analysis of the time-fractional heat conduction equation and its solution in the transform domain (26). The inverse sin-Fourier transform gives

$$
T^{*}(x, s)=T_{0} \frac{1}{s-i \omega} \exp \left(-\frac{x}{\sqrt{a}} s^{\alpha / 2}\right) .
$$

The inverse Laplace transform of $\exp \left(-\lambda s^{\alpha}\right)$ is expressed in terms of the Mainardi function $M(\alpha ; z)$ (see (A.11) from appendix). The solution has the form

$$
\begin{array}{r}
T(x, t)=\frac{\alpha T_{0} x}{2 \sqrt{a}} \int_{0}^{t} \frac{1}{\tau^{\alpha / 2+1}} M\left(\frac{\alpha}{2} ; \frac{x}{\sqrt{a} \tau^{\alpha / 2}}\right) e^{i \omega(t-\tau)}, \\
0<\alpha<2 .
\end{array}
$$

The particular case of the Mainardi function $M(1 / 2 ; z)$ is reduced to the exponential function (see $[48,49]$ ):

$$
M\left(\frac{1}{2} ; z\right)=\frac{1}{\sqrt{\pi}} \exp \left(-\frac{z^{2}}{4}\right),
$$

and solution (37) for $\alpha=1$ coincides with solution (31). have

Starting from the inverse Laplace transform of (26), we

$$
\widetilde{T}(\xi, t)=a T_{0} \xi \int_{0}^{t} \tau^{\alpha-1} E_{\alpha, \alpha}\left(-a \xi^{2} \tau^{\alpha}\right) e^{i \omega(t-\tau)} d \tau,
$$

where $E_{\alpha, \alpha}(z)$ is the Mittag-Leffler function in two parameters $\alpha$ and $\beta$ (see appendix). In parallel with (37), the inverse Fourier transform of (39) leads to another form of the solution

$$
\begin{aligned}
& T(x, t)=\frac{2 a T_{0}}{\pi} \\
& \quad \cdot \int_{0}^{t} \int_{0}^{\infty} \tau^{\alpha-1} E_{\alpha, \alpha}\left(-a \xi^{2} \tau^{\alpha}\right) e^{i \omega(t-\tau)} \xi \sin (x \xi) d \xi d \tau .
\end{aligned}
$$

Comparison of (37) and (40) allows us to establish the relation between the Mainardi function and the MittagLeffler function in the form of sin-Fourier transform (see also $[15,50]$, where the similar relations were obtained in terms of the cos-Fourier transform).

Figures 1 and 2 present the dependence of solution on distance in the case of the boundary condition

$$
T(x, t)=T_{0} \cos (\omega t) \quad x=0
$$

for different values of the order $\alpha$ of fractional derivative and different values of time. In numerical calculations we have used the following nondimensional quantities:

$$
\begin{aligned}
& \bar{T}=\frac{T}{T_{0}}, \\
& \bar{x}=\frac{x}{\sqrt{a} t^{\alpha / 2}}, \\
& \bar{t}=\omega t .
\end{aligned}
$$

To evaluate the Mittag-Leffler function $E_{\alpha, \alpha}(z)$, the algorithm suggested in the paper [51] has been used.

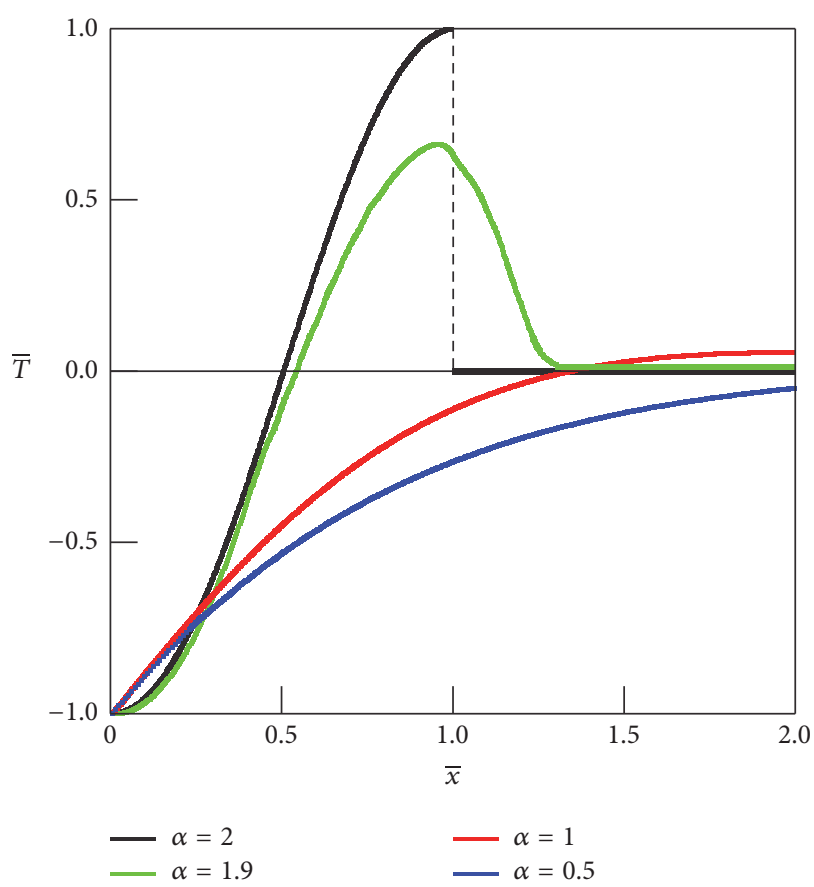

Figure 1: Dependence of temperature on distance $(\bar{t}=\pi)$.

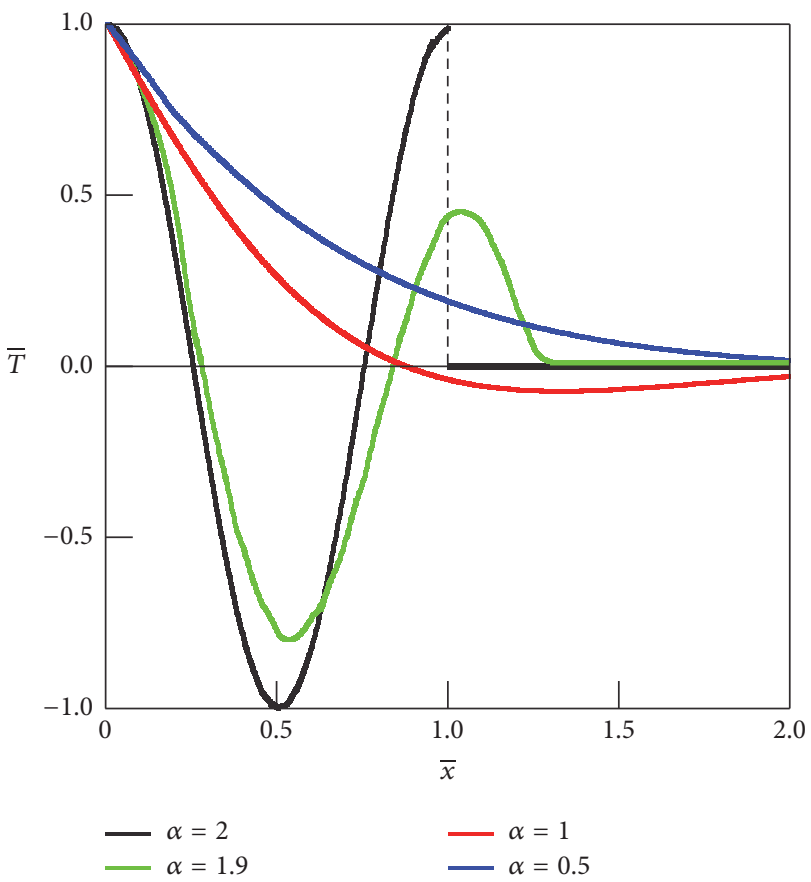

FIgURE 2: Dependence of temperature on distance $(\bar{t}=2 \pi)$.

\section{Concluding Remarks}

We have considered the Dirichlet problem for the timefractional heat conduction equation in a half-line with the Caputo fractional derivative and with the boundary value of temperature varying harmonically in time. The solution has been obtained using the integral transform technique. 
The Caputo fractional derivative of the exponential function has more complicated form than the corresponding derivative of the integer order. Hence, the Nowacki approach based on the representation of temperature as the product of a function of the spatial coordinate $U(x)$ and a function harmonic in time $e^{i \omega t}$ cannot be used, and the initial conditions should be taken into account.

In such a statement of the problem, the particular cases of the general solution for integer values of the order of derivative $(\alpha=1$ and $\alpha=2)$ describe both the quasisteady-state oscillations and the transient process. It should be emphasized that in the case of the ballistic heat conduction equation $(\alpha=2)$ the obtained solution presents the wavefront at $x=\sqrt{a} t(\bar{x}=1$ in Figures 1 and 2), which does not appear in the Nowacki-type solution.

The obtained solution may also be used in constructing solutions for boundary functions varying periodically in an arbitrary manner. Expanding the boundary function in the time-Fourier series, the solution can be obtained as a result of superposition of successive harmonic terms.

\section{Appendix}

We present integrals $[52,53]$ used in the paper:

$$
\begin{aligned}
& \int_{0}^{\infty} \frac{1}{x^{2}+c^{2}} \cos (b x) d x=\frac{\pi}{2 c} e^{-b c}, \\
& \int_{0}^{\infty} \frac{x}{x^{2}+c^{2}} \sin (b x) d x=\frac{\pi}{2} e^{-b c}, \quad b>0, \operatorname{Re} c>0 \\
& \int_{0}^{\infty} \frac{1}{x^{2}-c^{2}} \sin (b x) d x=\frac{\pi}{2} \cos (b c), \quad b>0, c>0, \\
& \int_{0}^{\infty} \frac{x}{x^{2}+c^{2}} e^{-a^{2} x^{2}} \sin (b x) d x=\frac{\pi}{4} \\
& \cdot e^{a^{2} x^{2}}\left[e^{-b c} \operatorname{erfc}\left(a c-\frac{b}{2 a}\right)\right. \\
& \left.-e^{b c} \operatorname{erfc}\left(a c+\frac{b}{2 a}\right)\right], \\
& \quad b>0, \operatorname{Re} a>0, \operatorname{Re} c>0
\end{aligned}
$$

where $\operatorname{erfc}(x)$ is the complementary error function

$$
\operatorname{erfc}(x)=\frac{2}{\sqrt{\pi}} \int_{x}^{\infty} e^{-t^{2}} d t
$$

Formulae for inverse Laplace transform (equations (A.6)-(A.10)) are borrowed from [54, 55]:

$$
L^{-1}\left\{\frac{1}{(s+a)(s+b)}\right\}=\frac{e^{-b t}-e^{-a t}}{a-b}
$$

$$
\begin{aligned}
L^{-1} & \left\{\frac{1}{(s+a)(\sqrt{s}+b)}\right\} \\
= & \frac{1}{a+b^{2}}\left[b e^{-a t}+\sqrt{a} e^{-a t} \operatorname{erfi}(\sqrt{a t})\right. \\
& \left.-b e^{b^{2} t} \operatorname{erfc}(b \sqrt{t})\right],
\end{aligned}
$$

where erfi $(z)$ is the error function of an imaginary argument:

$$
\begin{aligned}
\operatorname{erfi}(z) & =\frac{2}{\sqrt{\pi}} \int_{0}^{z} e^{t^{2}} d t, \\
L^{-1}\left\{\frac{e^{-c s}}{s+b}\right\} & = \begin{cases}e^{-b(t-c)}, & 0<c<t, \\
0, & 0<t<c,\end{cases} \\
L^{-1}\left\{e^{-\lambda \sqrt{s}}\right\} & =\frac{\lambda}{2 \sqrt{\pi} t^{3 / 2}} \exp \left(-\frac{\lambda^{2}}{4 t}\right) .
\end{aligned}
$$

Equation (A.11) can be found in $[15,48,49]$

$$
L^{-1}\left\{e^{-\lambda s^{\alpha}}\right\}=\frac{\alpha \lambda}{t^{\alpha+1}} M\left(\alpha ; \lambda t^{-\alpha}\right), \quad 0<\alpha<1, \lambda>0 .
$$

Here $M(\alpha ; z)$ is the Mainardi function [17, 48, 49], being the particular case of the Wright function:

$$
\begin{aligned}
& M(\alpha ; z)=\sum_{k=0}^{\infty} \frac{(-1)^{k} z^{k}}{k ! \Gamma[-\alpha k+(1-\alpha)]} \\
& \\
& 0<\alpha<1, z \in C .
\end{aligned}
$$

Equation (A.13) is taken from $[16,17]$

$$
L^{-1}\left\{\frac{s^{\alpha-\beta}}{s^{\alpha}+b}\right\}=t^{\beta-1} E_{\alpha, \beta}\left(-b t^{\alpha}\right),
$$

where $E_{\alpha, \beta}(z)$ is the Mittag-Leffler function in two parameters $\alpha$ and $\beta[16,17,56]$ described by the following series representation:

$$
E_{\alpha, \beta}(z)=\sum_{k=0}^{\infty} \frac{z^{k}}{\Gamma(\alpha k+\beta)}, \quad \alpha>0, \beta>0, z \in C .
$$

\section{Competing Interests}

The author declares that there is no conflict of interests regarding the publication of this paper.

\section{References}

[1] W. Nowacki, "State of stress in an elastic space due to a source of heat varying harmonically as function of time," Bulletin de l'Académie Polonaise des Sciences, Série des Sciences Techniques, vol. 5, no. 3, pp. 145-154, 1957.

[2] W. Nowacki, Thermoelasticity, Poland and Pergamon Press, Oxford, UK, 2nd edition, 1986.

[3] T. L. Bergman, A. S. Lavine, F. P. Incropera, and D. P. DeWitt, Fundamentals of Heat and Mass Transfer, John Wiley \& Sons, Hoboken, NJ, USA, 7th edition, 2011. 
[4] C. Cattaneo, "Sulla conduzione del calore," Atti del Seminario Matematico e Fisico dell' Universitè di Modena, vol. 3, no. 3, pp. 83-101, 1948.

[5] M. E. Gurtin and A. C. Pipkin, "A general theory of heat conduction with finite wave speeds," Archive for Rational Mechanics and Analysis, vol. 31, no. 2, pp. 113-126, 1968.

[6] D. D. Joseph and L. Preziosi, "Heat waves," Reviews of Modern Physics, vol. 61, no. 1, pp. 41-73, 1989.

[7] A. E. Green and P. M. Naghdi, "Thermoelasticity without energy dissipation," Journal of Elasticity, vol. 31, no. 3, pp. 189-208, 1993.

[8] R. Metzler and J. Klafter, "The random walk's guide to anomalous diffusion: a fractional dynamics approach," Physics Reports, vol. 339, no. 1, pp. 1-77, 2000.

[9] R. Metzler and J. Klafter, "The restaurant at the end of the random walk: recent developments in the description of anomalous transport by fractional dynamics," Journal of Physics A: Mathematical and General, vol. 37, no. 31, pp. R161-R208, 2004.

[10] J. Ignaczak and M. Ostoja-Starzewski, Thermoelasticity with Finite Wave Speeds, Oxford University Press, Oxford, UK, 2009.

[11] Y. Z. Povstenko, "Fractional heat conduction equation and associated thermal stress," Journal of Thermal Stresses, vol. 28, no. 1, pp. 83-102, 2005.

[12] Y. Povstenko, Fractional Thermoelasticity, Springer, New York, NY, USA, 2015.

[13] Y. Povstenko, "Thermoelasticity which uses fractional heat conduction equation," Journal of Mathematical Sciences, vol. 162, no. 2, pp. 296-305, 2009.

[14] Y. Povstenko, "Non-axisymmetric solutions to time-fractional diffusion-wave equation in an infinite cylinder," Fractional Calculus and Applied Analysis, vol. 14, no. 3, pp. 418-435, 2011.

[15] Y. Povstenko, Linear Fractional Diffusion-Wave Equation for Scientists and Engineers, Birkhäuser, New York, NY, USA, 2015.

[16] R. Gorenflo and F. Mainardi, "Fractional calculus: integral and differential equations of fractional order," in Fractals and Fractional Calculus in Continuum Mechanics, A. Carpinteri and F. Mainardi, Eds., vol. 378 of CISM Courses and Lectures, pp. 223-276, Springer, Vienna, Austria, 1997.

[17] I. Podlubny, Fractional Differential Equations, Academic Press, New York, NY, USA, 1999.

[18] A. A. Kilbas, H. M. Srivastava, and J. J. Trujillo, Theory and Applications of Fractional Differential Equations, Elsevier, Amsterdam, The Netherlands, 2006.

[19] R. Gorenflo and F. Mainardi, "Fractional calculus and stable probability distributions," Archives of Mechanics, vol. 50, no. 3, pp. 377-388, 1998.

[20] F. Mainardi and R. Gorenflo, "On Mittag-Leffler-type functions in fractional evolution processes," Journal of Computational and Applied Mathematics, vol. 118, no. 1-2, pp. 283-299, 2000.

[21] F. Mainardi, Fractional Calculus and Waves in Linear Viscoelasticity: An Introduction to Mathematical Models, Imperial College Press, London, UK, 2010.

[22] Yu. N. Rabotnov, Creep Problems in Structural Members, NorthHolland Publishing Company, Amsterdam, The Netherlands, 1969.

[23] K. B. Oldham and J. Spanier, The Fractional Calculus, Academic Press, New York, NY, USA, 1974.

[24] Y. A. Rossikhin and M. V. Shitikova, "Applications of fractional calculus to dynamic problems of linear and nonlinear hereditary mechanics of solids," Applied Mechanics Reviews, vol. 50, no. 1, pp. 15-67, 1997.
[25] G. M. Zaslavsky, "Chaos, fractional kinetics, and anomalous transport," Physics Reports, vol. 371, no. 6, pp. 461-580, 2002.

[26] B. J. West, M. Bologna, and P. Grigolini, Physics of Fractal Operators, Institute for Nonlinear Science, Springer, New York, NY, USA, 2003.

[27] R. L. Magin, Fractional Calculus in Bioengineering, Begell House, Connecticut, Mass, USA, 2006.

[28] Y. A. Rossikhin and M. V. Shitikova, "Application of fractional calculus for dynamic problems of solid mechanics: novel trends and recent results," Applied Mechanics Reviews, vol. 63, no. 1, Article ID 010801, 52 pages, 2010.

[29] V. Gafiychuk and B. Datsko, "Mathematical modeling of different types of instabilities in time fractional reaction-diffusion systems," Computers \& Mathematics with Applications, vol. 59, no. 3, pp. 1101-1107, 2010.

[30] V. V. Uchaikin, Fractional Derivatives for Physicists and Engineers, Springer, Berlin, Germany, 2013.

[31] G. M. Zaslavsky, Hamiltonian Chaos and Fractional Dynamics, Oxford University Press, New York, NY, USA, 2005.

[32] V. E. Tarasov, Fractional Dynamics: Applications of Fractional Calculus to Dynamics of Particles, Fields and Media, Higher Education Press, Bejing, China; Springer, Berlin, Germany, 2010.

[33] J. S. Leszczyński, An Introduction to Fractional Mechanics, The Publishing Office of Czestochowa University of Technology, Czestochowa, Poland, 2011.

[34] D. Baleanu, J. A. Tenreiro Machado, and A. C. J. Luo, Eds., Fractional Dynamics and Control, Springer, New York, NY, USA, 2012.

[35] T. M. Atanacković, S. Pilipović, B. Stanković, and D. Zorica, Fractional Calculus with Applications in Mechanics: Vibrations and Diffusion Processes, John Wiley \& Sons, Hoboken, NJ, USA, 2014.

[36] V. Uchaikin and R. Sibatov, Fractional Kinetics in Solids: Anomalous Charge Transport in Semiconductors, Dielectrics and Nanosystems, World Scientific, New Jersey, NJ, USA, 2013.

[37] R. Herrmann, Fractional Calculus: An Introduction for Physicists, World Scientific, Singapore, 2nd edition, 2014.

[38] R. Abi Zeid Daou and X. Moreau, Eds., Fractional Calculus: Applications, NOVA Science Publishers, New York, NY, USA, 2015.

[39] Y. Z. Povstenko, "Fractional Cattaneo-type equations and generalized thermoelasticity," Journal of Thermal Stresses, vol. 34, no. 2, pp. 97-114, 2011.

[40] A. S. El-Karamany and M. A. Ezzat, "On fractional thermoelasticity," Mathematics and Mechanics of Solids, vol. 16, no. 3, pp. 334-346, 2011.

[41] M. A. Ezzat and M. A. Fayik, "Fractional order theory of thermoelastic diffusion," Journal of Thermal Stresses, vol. 34, no. 8, pp. 851-872, 2011.

[42] Y. Povstenko, "Harmonic impact in the plane problem of fractional thermoelasticity," in Proceedings of the 11th International Congress on Thermal Stresses, pp. 227-230, Salerno, Italy, June 2016.

[43] Y. Povstenko, "Fractional heat conduction in a space with a source varying harmonically in time and associated thermal stresses," Journal of Thermal Stresses, vol. 39, no. 11, pp. 14421450, 2016.

[44] M. Abramowitz and I. A. Stegun, Eds., Handbook of Mathematical Functions with Formulas, Graphs and Mathematical Tables, New York, NY, USA, Dover, 1972. 
[45] R. R. Nigmatullin, "The realization of the general transfer equation in a medium with fractal geometry," Physica Status Solidi B, vol. 133, no. 1, pp. 425-430, 1986.

[46] V. E. Arkhincheev, "Anomalous diffusion and charge relaxation on comb model: Exact solutions," Physica A: Statistical Mechanics and its Applications, vol. 280, no. 3-4, pp. 304-314, 2000.

[47] V. E. Arkhincheev, "Diffusion on random comb structure: effective medium approximation," Physica A: Statistical Mechanics and Its Applications, vol. 307, no. 1-2, pp. 131-141, 2002.

[48] F. Mainardi, "The fundamental solutions for the fractional diffusion-wave equation," Applied Mathematics Letters, vol. 9, no. 6, pp. 23-28, 1996.

[49] F. Mainardi, "Fractional relaxation-oscillation and fractional diffusion-wave phenomena," Chaos, Solitons and Fractals, vol. 7, no. 9, pp. 1461-1477, 1996.

[50] Y. Povstenko, "Generalized theory of diffusive stresses associated with the time-fractional diffusion equation and nonlocal constitutive equations for the stress tensor," Computers \& Mathematics with Applications, 2016.

[51] R. Gorenflo, J. Loutchko, and Y. Luchko, "Computation of the Mittag-Leffler function and its derivatives," Fractional Calculus and Applied Analysis, vol. 5, no. 4, pp. 491-518, 2002.

[52] A. P. Prudnikov, Y. A. Brychkov, and O. I. Marichev, Integrals and Series, Vol. 1: Elementary Functions, Gordon and Breach Science, Amsterdam, The Netherlands, 1986.

[53] A. Erdélyi, W. Magnus, F. Oberhettinger, and F. G. Tricomi, Tables of Integral Transforms, vol. 1, McGraw-Hill, New York, NY, USA, 1954.

[54] A. P. Prudnikov, Yu. A. Brychkov, and O. I. Marichev, Integrals and Series, Vol. 5: Inverse Laplace Transforms, vol. 5, Gordon and Breach Science Publishers, Amsterdam, The Netherlands, 1992.

[55] V. A. Ditkin and A. P. Prudnikov, Reference Book on Operational Calculus, Higher School, Moscow, Russia, 1965 (Russian).

[56] R. Gorenflo, A. A. Kilbas, F. Mainardi, and S. V. Rogosin, MittagLeffler Functions, Related Topics and Applications, Springer, Berlin, Germany, 2014. 


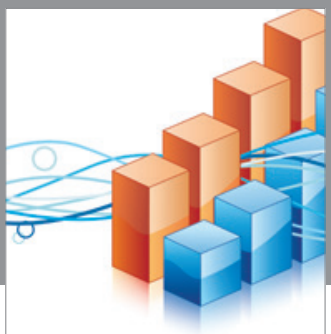

Advances in

Operations Research

vatem alat4

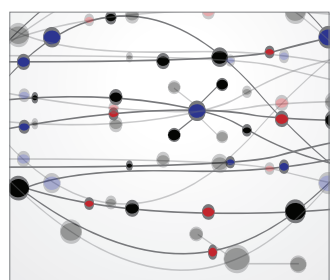

\section{The Scientific} World Journal
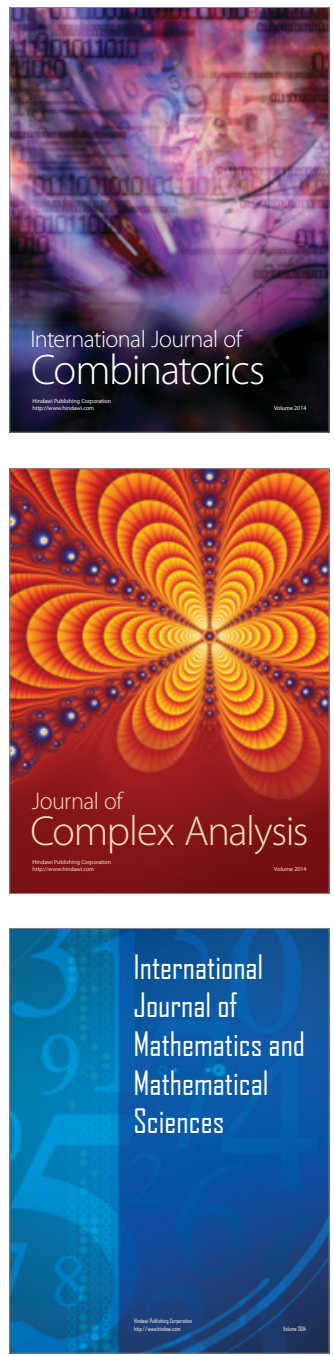
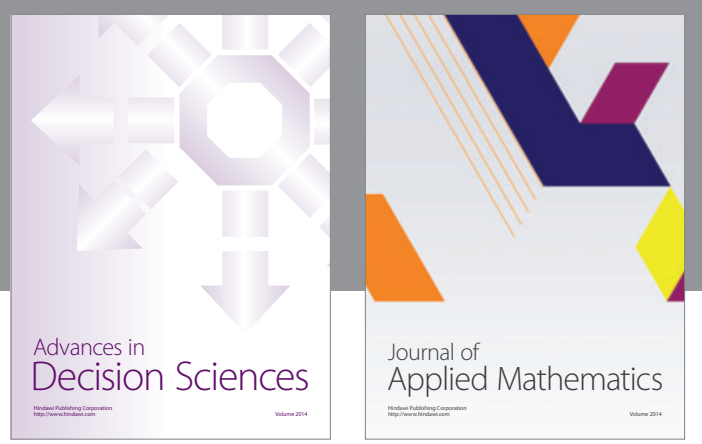

Algebra

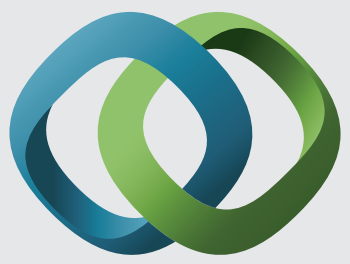

\section{Hindawi}

Submit your manuscripts at

http://www.hindawi.com
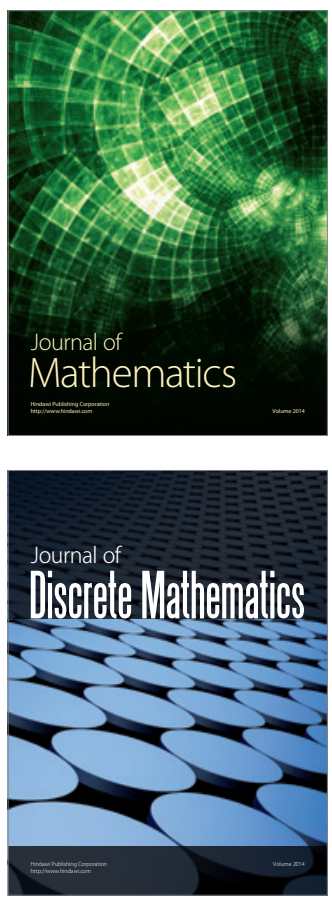

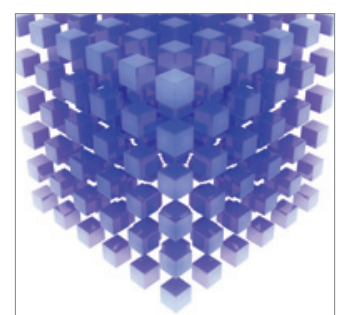

Mathematical Problems in Engineering
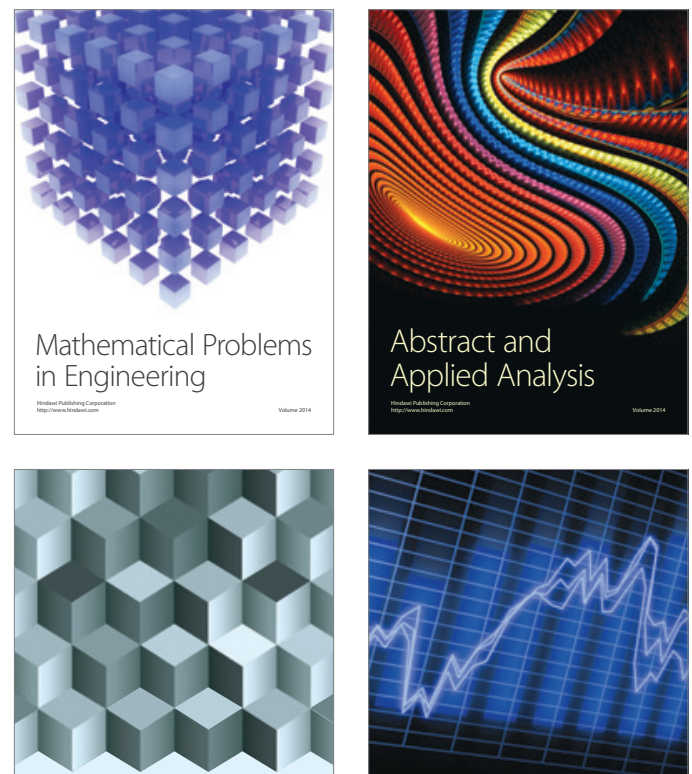

Journal of

Function Spaces

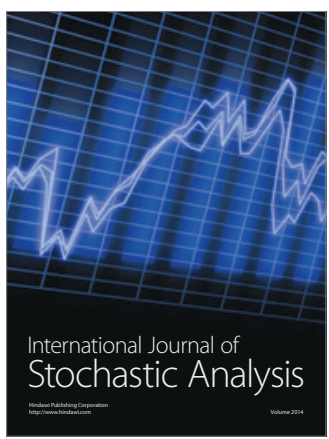

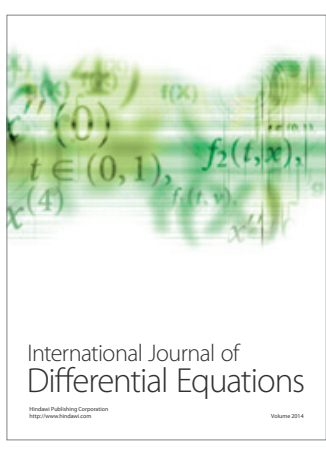
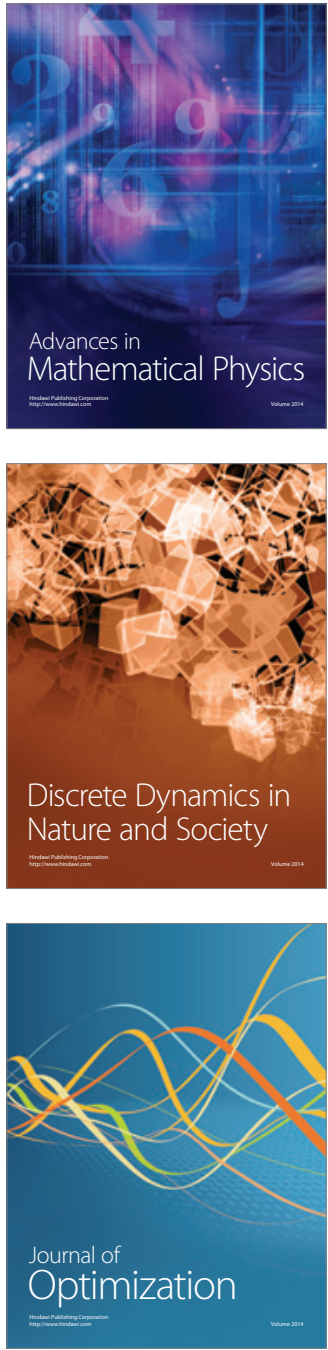